\title{
Diffractometer for High Energy X-rays at the APS
}

U. Ruett', M. A. Beno ${ }^{a}$, J Strempfer ${ }^{b}$, G. Jennings ${ }^{a}$, C. Kurtz ${ }^{a}$, and Pedro A. Montano $o^{a, c}$

${ }^{2}$ Materials Science Division and Basic Energy Sciences Synchrotron Radiation Center, Argonne National Laboratory, Argonne, IL 60439, USA

${ }^{b}$ Department of Physics, Northern Illinois University, DeKalb, IL 60115

'Department of Physics, University of Illinois at Chicago, Chicago, ㅍ, USA

Materials Science Division
Argonne National Laboratory
Argonne, IL 60439
$\begin{aligned} & \text { The submitted manuscript has been created by } \\ & \text { the University of Chicago as Operator of Argonne } \\ & \text { National Laboratory ("Argonne") under Contract } \\ & \text { No. W-31-109-ENG-38 with the U.S. Department } \\ & \text { of Energy. The U.S. Government retains for } \\ & \text { itself, and others acting on its behalf, a paid-up. } \\ & \text { non exclusive, irrevocable worldwide license in } \\ & \text { said article to reproduce, prepare derivative } \\ & \text { works, distribute copies to the public, and } \\ & \text { perform publicly and display publicly, by or on } \\ & \text { behalf of the Govemment. }\end{aligned}$

\section{September 2000}

/jc

Distribution:

1-2. PRS, 203

3. J. M. Gibson

4. P. A. Montano

5. F. Y. Fradin

6. Office of Science

7. J. Coble

8. Authors

Submitted for publication in Nuclear Instrument and Methods, Section A, from the $7^{\text {th }}$ International Synchrotron Radiation Instrumentation conference (SRI 2000) held August 20-25 in Berlin, Germany.

This work is supported by the US Department of Energy, Office of Basic Energy Sciences, under contract No. W-31-109-ENG-38. 


\section{DISCLAIMER}

This report was prepared as an account of work sponsored by an agency of the United States Government. Neither the United States Government nor any agency thereof, nor any of their employees, make any warranty, express or implied, or assumes any legal liability or responsibility for the accuracy, completeness, or usefulness of any information, apparatus, product, or process disclosed, or represents that its use would not infringe privately owned rights. Reference herein to any specific commercial product, process, or service by trade name, trademark, manufacturer, or otherwise does not necessarily constitute or imply its endorsement, recommendation, or favoring by the United States Government or any agency thereof. The views and opinions of authors expressed herein do not necessarily state or reflect those of the United States Government or any agency thereof. 


\section{DISCLAIMER}

Portions of this document may be illegible in electronic image products. Images are produced from the best available original document. 


\title{
Diffractometer for High Energy X-rays at the APS
}

\author{
U. Rütt ${ }^{a}$, M.A. Beno ${ }^{a}$, J. Strempfer ${ }^{b}$, G. Jennings ${ }^{a}$, C. Kurtz ${ }^{a}$ and P.A. Montano ${ }^{a, c}$ \\ ${ }^{a}$ Materials Science Division and Basic Energy Sciences Synchrotron Radiation \\ Center at Argonne National Laboratory, Argonne, IL 60439; USA \\ ${ }^{6}$ Department of Physics, Northern Illinois University, DeKalb, IL 605115, USA \\ 'Department of Physics, University of Illinois at Chicago, Chicago, IL, USA
}

Corresponding author: U. Rütt, MSD 433-E-003, Argonne National Laboratory, 9700 S. Cass Ave, Argonne, IL 60439, USA. Tel. (630) 252 0383, Fax:

(630) 252 0365, email: ruett@msd.anl.gov

Keywords: high energy $\mathrm{X}$-rays, synchrotron radiation, diffraction

PACS0.7.85.Qe

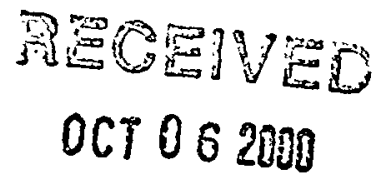

연.

\begin{abstract}
The Basic Energy Sciences Synchrotron Radiation Center Collaborative Access Team (BESSRC-CAT) has designed and built a diffractometer specialized for high energy synchrotron radiation (E> $60 \mathrm{keV}$ ) at the Advanced Photon Source (APS). The diffractometer, which is installed at the elliptical multipole wiggler, uses linearly polarized light [1]. The instrument is a triple-axis diffractometer allowing a high resolution measurement in two dimensions of the reciprocal space. As opposed to the other diffractometers for high photon energies at the HASYLAB (Germany) and ESRF (France) $[2,3]$, this diffractometer utilizes the vertical scattering plane to take full advantage of the small vertical divergence of the beam and to allow horizontal focusing of the broad beam from the wiggler without disturbing the resolution of the instrument. The instrument is designed to carry heavy sample equipment up to a weight of $200 \mathrm{~kg}$, while maintaining high resolution and low background. First tests have been done to check the overall performance, stability, background and resolution of the diffractometer.
\end{abstract}


Experiments using high energy synchrotron radiation $(\mathrm{E}>60 \mathrm{keV})$ gain from a large mean free beam path of the photons in materials, because the energy is above the $\mathrm{K}$ edges of most elements. At high photon energies momentum transfers of $Q>45 \AA^{-1}$ are easily achievable. In addition the correction factors due to polarization, absorption and extinction are in general small [1,2,3]. A special application is non-resonant magnetic scattering, where at high $\mathrm{X}$-ray energies the magnetic cross-section is proportional to the square of the spin component perpendicular to the scattering plane [4]. This allows the determination of the pure spin contribution to the magnetic moment [5]. The high penetration into material and the high momentum transfers make high energy photons comparable to neutron experiments, but with the high resolution and high flux of modern $\mathrm{X}$-ray instruments at synchrotron radiation sources.

A dedicated triple-axis diffractometer consisting of monochromator, sample and analyzer provides high $\mathrm{q}$-space resolution in two dimensions. The monochromator crystal determines the scattering plane and the energy bandwidth. The sample rotation corresponds to a motion perpendicular to the scattering vector of the sample in reciprocal space. The analyzer rotation determines the observed lattice spacing at the sample and allows studies in two dimensions of the reciprocal space. For three perfect Si 220 crystals at $110 \mathrm{keV}$ photon energy the q-space resolution defined by the FWHM is $10^{-5} \AA^{-1}$ and $210^{-4} \AA^{-1}$ perpendicular and parallel to the reciprocal lattice vector. In angular space this corresponds to 0.6 arcsec. The resolution perpendicular to the scattering plane is determined by the horizontal slit openings and the distance between sample and detector. Because of low absorption and small Bragg angles all crystals are 
used in transmission geometry. This allows studies of large samples (up to several mm) even in heavy sample environment.

If enough flexibility is provided, the diffractometer can also be used for powder diffraction gaining from good averaging over a large sample volume as well as for studies of pair distribution functions in amorphous or powdered materials, which take full advantage of large momentum transfers [2].

The Basic Energy Sciences Synchrotron Radiation Center (BESSRC) has built a triple axis diffractometer for high energy photons at the elliptical multipole wiggler (EMW) at the Advanced Photon Source (APS). The wiggler with a critical photon energy of 32 $\mathrm{keV}$ serves three experimental stations simultaneously [6], two with linearly polarized light and one with circularly polarized light. The high energy station uses linearly polarized light, which is deflected horizontally by a pre-monochromator at a Bragg angle of $\theta=1.9^{\circ}$ into the station. The pre-monochromator, which can accept up to 0.33 mrad of the horizontal beam divergence, is at distance of $32 \mathrm{~m}$ from the source and 22 m away from the diffractometer. If an annealed Silicon 220 crystal with a mosaicity of 10 arcsec [7] is used in transmission geometry, the beam spot of $98 \mathrm{keV}$ photons at the diffractometer is up to $3.5 \mathrm{~mm}$ high and $2.5 \mathrm{~mm}$ wide with an intensity of $210^{12}$ photons/sec and an energy resolution of $\Delta \mathrm{E} / \mathrm{E}=0.01$. The beam size and energy band width can be adapted by a slit directly behind the pre-monochromator. For high resolution experiments only $0.06 \mathrm{mrad}$ of the beam would be scattered providing an energy bandwidth of $\Delta E / E=0.002$. The beam spot in the hutch is hereby reduced to 3.5 $\mathrm{mm}$ vertically and about $1 \mathrm{~mm}$ horizontally with an intensity of $410^{11}$ photons $/ \mathrm{sec}$. An 
annealed bent Bragg crystal could accept 0.33 mrad beam divergence with an energy resolution of $\Delta \mathrm{E} / \mathrm{E}=0.002$ providing a spot size of $3.5 \times 1 \mathrm{~mm}^{2}$ with $410^{12}$ photons $/ \mathrm{sec}$. It is planned to switch to this optic next year.

The photon energy can only be changed by the choice of the lattice spacing of the premonochrometer. At the moment annealed Si220, Si111 and Si311 crystals are available scattering $60 \mathrm{keV}, 98 \mathrm{keV}$ and $115 \mathrm{keV}$ into the hutch, respectively.

As opposed to the three dedicated triple crystal diffractometers for high energy photons in Europe (two at HASYLAB, Germany and one at the ESRF, France) this diffractometer is operating in the vertical scattering plane, see Fig. 1[2,3]. The disadvantage of this geometry were the difficulties in the design to allow for heavy sample environment while maintaining the angular stability required for high resolution experiments. The advantage is the flexibility to use it for high momentum transfers as well as for high resolution experiments and the option of focussing in the horizontal scattering plane while taking advantage of the narrow vertical beam divergence for high energy resolution.

The diffractometer has four towers for monochromator, sample, analyzer and detector. The first three are placed on a common optical table (Newport RS4000, $3.6 \mathrm{~m}$ long, 1.5 $\mathrm{m}$ wide and $203 \mathrm{~mm}$ thick). The monochromator and analyzer tower are $610 \mathrm{~mm}$ long, the sample tower $650 \mathrm{~mm}$ and $1.5 \mathrm{~m}$ broad. All three towers can be moved along the table on a rail system. Monochromator and analyzer tower are equipped with a Huber 420 goniometer for the theta-rotation and a Huber 408 and Huber 5202.5 circle segment for the crystal alignment. On the analyzer tower the theta-rotation is controlled by a Heidenhain encoder (RON 905 with AWE1024, 0.2 arcsec accuracy and 0.04 arcsec step resolution). The goniometer can be translated by a vertical (z) rail system ( $900 \mathrm{~mm}$ 
travel). An additional horizontal (x) rail system below this tower gives the option to scatter horizontally with the diffractometer. The sample tower has a heavy duty ztranslation as the base of the rotation tables. The theta-rotation is realized by a Huber 430 with counter point which supports the "swing" on which the following tables are built: a Huber 420 as "psi"-rotation, a circle segment made by Huber with an angular range of $+/-10^{\circ}$ providing the chi-rotation, a Schneeberger z-translation $(+/-12 \mathrm{~mm})$ and $\mathrm{x}$-translation $(+/-50 \mathrm{~mm})$. Optional an additional Huber 420 as phi-rotation parallel to the theta-rotation or a kappa-arm under an angle of 60 degree can be mounted. The translations allow the choice of different sample volumes in large crystals. The sample tower is designed to be able to carry heavy loads up to $200 \mathrm{~kg}$. All three theta-rotations are monitored by tilt sensors (Applied Geomechanics, model 781) to control unwanted angular motions (better than $0.1 \mathrm{arcsec}$ )

The detector tower is located behind the optical table on three rail systems. The distance between detector tower and optical table can be varied between 0 and $1.5 \mathrm{~m}$. The longest possible distance between sample tower and detector is more than $3 \mathrm{~m}$. A $\mathrm{y}$-translation, which allows the use of the horizontal scattering plane is $2 \mathrm{~m}$ long, the $\mathrm{z}$ translation has a travel range of $1.2 \mathrm{~m}$ (without way covers $1.5 \mathrm{~m}$ ). The detector is rotated by a Huber 410 supported by a counterpoint keeping it parallel to the incident beam. The detector is a single element Germanium solid state detector with a $18 \mathrm{~mm}$ thick and $16 \mathrm{~mm}$ in diameter crystal. Its energy resolution is better than $700 \mathrm{eV}$ at 122 $\mathrm{keV}$ with $1 \mu \mathrm{sec}$ shaping time. The 131 dewar for the liquid nitrogen lasts for 7 days. The utilization of monochromator and analyzer crystals are optional and are only needed for high resolution experiments. An analyzer with a broad mosaicity, e.g. 
annealed silicon, can also function like a narrow slit to suppress background, even if good two dimensional resolution is not required.

For experiments with powders or amorphous materials studying pair correlation functions the sample can be placed on the analyzer tower. In this case a $\mathrm{x}$ - and $\mathrm{z}-$ translation are available for an easy sample alignment. Sample and detector can be as close as $650 \mathrm{~mm}$. A momentum transfer of $48 \AA^{-1}$ can be reached using $115 \mathrm{keV}$ photon energy.

Between the four towers are optical rails guided automatically with the $\mathrm{z}$-translations of the towers. On these rails motorized slit systems, collimators and beam monitors can be placed. As slit systems standard Huber slits and two special KOHZU slits are available. The KOHZU slits are equipped with $5 \mathrm{~mm}$ tungsten and $10 \mathrm{~mm}$ tantalum blades, respectively. The collimators are made out of brass. They have holes for the beam path of $10 \mathrm{~mm}$ or $20 \mathrm{~mm}$ in diameter. As beam monitors simple silicon photon diodes (10 $\mathrm{mm} \times 10 \mathrm{~mm} \times 1 \mathrm{~mm}$ thick) are used in transmission geometry. The current induced by the beam is amplified and converted to voltage. The voltage is converted into frequency and read out by a scaler card.

As sample surroundings a Helium flow cryostat and a displex are available at the moment for low temperature work. In general no special windows are necessary because of the small absorption of the high energy photons, so that various laboratory equipment could be used.

The user interface to the diffractometer is implemented in the program package Igor running on Macintosh. Motors and scalers are driven by a VME-crate with EPICS system. UB-matrix can be used to perform scans in the reciprocal space.

of the towers. 
To test the stability of the instrument, perfect Si 220 crystals were mounted on the monochromator and analyzer tower. The analyzer crystal was scanned over $12 \mathrm{~h}$, with a measurement done every 10 min to check for changes in the angle. As shown in Fig. 2, the stability was better than 0.15 arcsec, when the tilt sensor were taken into account. Over long term the stability increased, supposedly because the instrument was undisturbed during the measurement.

A further test of the reliability of the diffractometer was the determination of absolute lattice constants with a reference crystal [8]. For this experiment three perfect Si220 crystals were used as monochromator, reference sample and analyzer crystals. In addition, the sample (here Ge220) was placed next to the reference crystal on the sample stage. First a precise determination of the incident photon wavelength $\lambda$ is needed. Using transmission geometry, $\lambda=0.12588 \AA$ was calculated by the rotation angle between the $\mathrm{Si}(220)$ and $\mathrm{Si}(-2-20)$ reflection of the analyzer crystal. The angle of the analyzer crystal is a measure for the lattice constant of the sample. It was first aligned to the reflection of the reference crystal with a well known lattice spacing $\mathrm{d}_{\text {ref }}=$ $1.92011 \AA$ and then to the sample crystal with the unknown lattice spacing $d_{\text {sample }}$. From the difference in the angle $\Delta \omega=542.6$ arcsec of the analyzer crystal for the two reflections, the lattice constant of the sample can be calculated by $d_{\text {sample }}=\mathrm{d}_{\text {ree }} f(1-\Delta \omega \cdot$ $\mathrm{d}_{\mathrm{ref}}(\lambda)=2.00038 \AA$. Assuming we can measure $\Delta \omega$ better than 1 arcsec, an absolute lattice spacing can be determined to a precision $0.01 \%$ of the lattice spacing of the reference crystal with $\lambda$ in the order of $0.1 \AA$. In this test we obtained a lattice constant of $\mathrm{a}=5.6579 \AA$ for Germanium, the literature value is $\mathrm{a}=5.6575 \AA$. 
Acknowledgement: Work at Argonne National Laboratory is supported by the Department of Energy (DOE), Office of Basic Energy Sciences, Division of Material Sciences, under contract W-31-109-ENG-38 and the State of Illinois under HECA. The authors would like to thank J.R. Schneider for helpful discussions and advice. 


\section{References}

[1] U. Rütt, J.R. Schneider, M.A. Beno, G.S. Knapp and P.A. Montano (1998), Proc. SPIE 3348, 132.

[2] R. Bouchard, D. Hupfeld, T. Lippmann, J. Neuefeind, H.-B. Neumann, H.F. Poulsen, U. Rütt, T. Schmidt, J. R. Schneider, J. Süßenbach and M. v. Zimmermann (1998), J. Synchrotron Rad. 5, 90

[3] K.-D. Liss, A. Royer, T. Tschentscher, P. Suortti and A.P. Williams (1998), J. Synchrotron Rad. 5, 82

[4] M. Lippert, T. Brückel, T. Köhler and J.R. Schneider (1994), Europhys. Lett. 27,537

[5] J. Strempfer, T. Brückel, D. Hupfeld, J.R. Schneider, K.D. Liss and T. Tschentscher, (1997) Europhys. Lett. 40, 569

[6] M.A. Beno, C. Kurtz, A. Munkholm, U. Rütt, M. Engbretson, G. Jennings, J. Linton, G.S. Knapp and P.A. Montano.'Elliptical Multipole Wiggler Beamlines at the Advanced Photon Source", these proceedings.

[7] S. Keitel, U. Rütt, M.A. Beno and J.R. Schneider, "Optimization of monochromator crystals by in situ studies of the annealing process of Cz-grown silicon". (1998) HASYLAB Annual Report

[8] W.L.Bond, Acta Cryst 13, 814 (1960) 


\section{Figure captions}

1.Sketch of the diffractometer. The three towers for the crystals are mounted together on an optical table $(3.6 \mathrm{~m} \times 1.5 \mathrm{~m})$. The detector is placed behind the diffractometer on a tower that can be translated in all three direction. The detector is kept parallel to the incoming beam by a rotation. Optical rails between the towers are optical allow mounting of slit systems, beam collimators and beam monitors. For a more detailed description see text.

2.Test of the angular stability. Perfect Si 220 crystals were mounted on the monochromator and analyzer tower. The analyzer crystal was scanned every 10 minutes with its rotation controlled by a Heidenhain encoder. In addition the stability of the towers was monitored by tilt sensors. The peak position of the analyzer reflection was stable $<0.3$ arcsec over $12 \mathrm{~h}$. After the correction for the motion of the towers from the tilt sensor, the stability was better than 0.15 arcsec. 


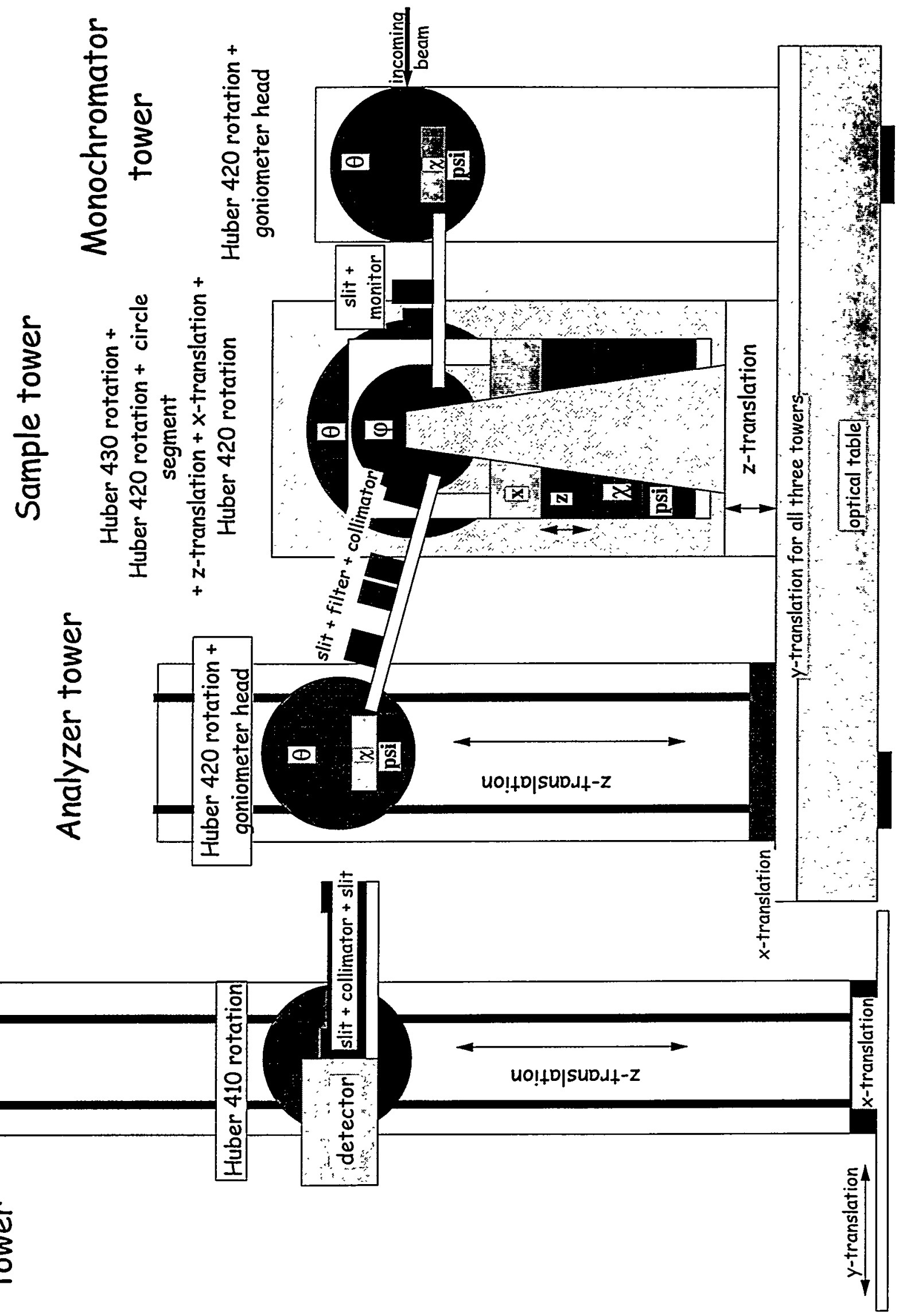




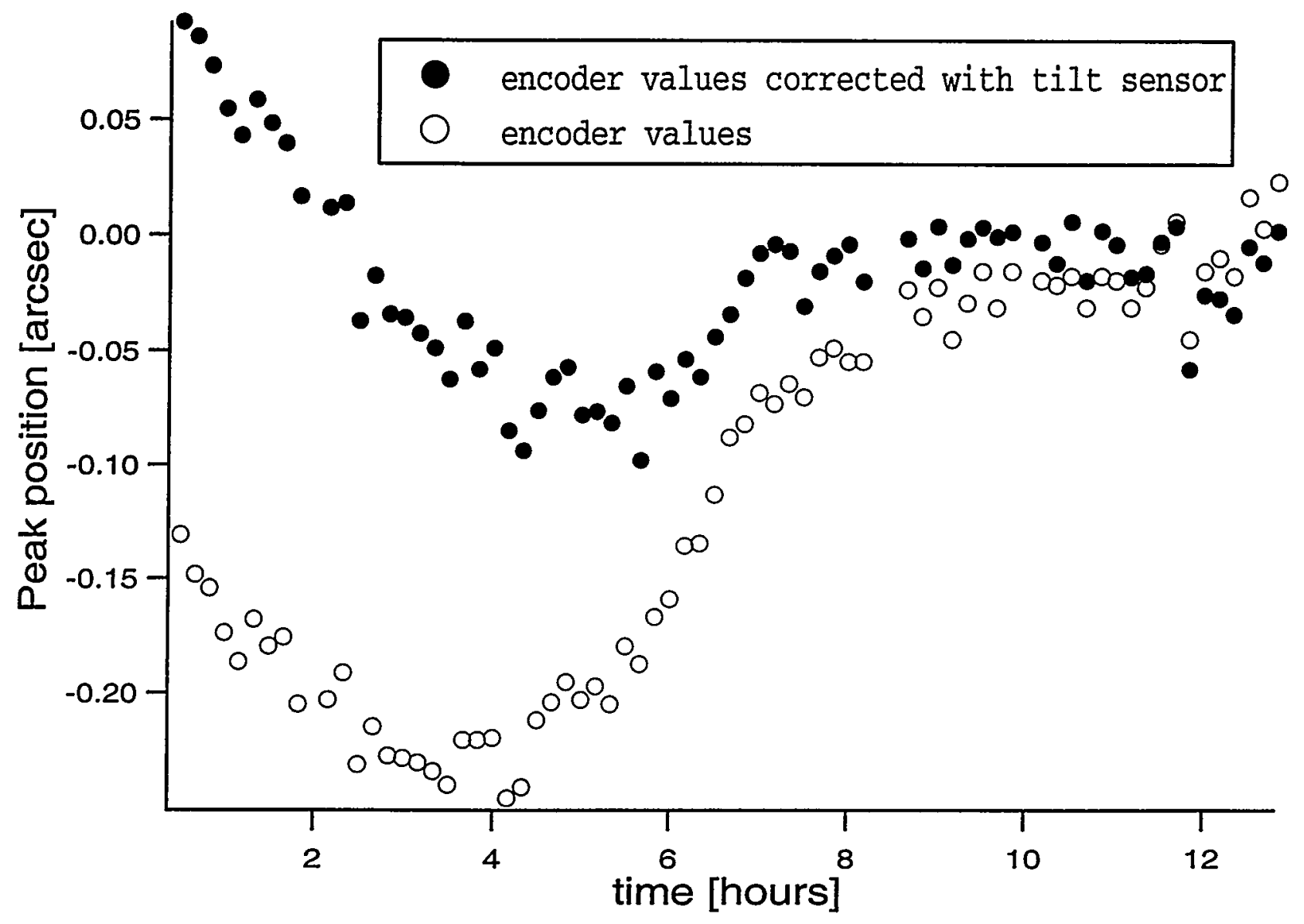

Fis: 